\title{
Influence of different dietary protein levels and feeding frequencies on growth performance and feed utilization of the Giant freshwater prawn, Macrobrachium rosenbergii with the Nile tilapia, Oreochromus niloticus in polyculture
}

\author{
Ashraf M. A. S. Goda'; Mohamed I. A-H Wafaa ${ }^{1}$; Eglal A. Omar ${ }^{2}$; \\ Nagy M. El-Bermawey ${ }^{2}$ and Shaimaa M.A. Hebalah ${ }^{1}$ \\ 1- Fish Nutrition Laboratory, National Institute of Oceanography and \\ Fisheries (NIOF), Egypt.
}

2- Department of Animal and Fish Production, Faculty of Agriculture (Saba Basha), Alexandria University, Egypt.

\begin{abstract}
A n 84-day experiment was conducted to investigate the effects of two dietary protein levels $30 \& 35 \% \mathrm{CP} \&$ three feeding frequencies $2,3 \& 4$ times a day $(8: 0$ and $11.0 \mathrm{~h}),(8.0,11.0$ and $14.0 \mathrm{~h})$ and $(8.0,11.0,14.0$ and $17.0 \mathrm{~h})$ on growth performance and feed utilization of the freshwater prawn, Macrobrachium rosenbergii with the Nile tilapia, Oreochromuis niloticus fry in a polyculture system. The experiment was conducted in small-scale hapas, 3.75 $\mathrm{m}^{-3}$ each in triplicates. Irrespective of polyculture system, prawn PL fed diet containing $35 \%$ CP 4 times a day had significant $(\mathrm{P} \leq 0.05)$ the highest final body weight (FBW), weight gain (WG), specific growth rate(SGR) and best feed conversion ratio (FCR) values among all treatments. Meanwhile, the Nile tilapia fed on diet containing 35\% CP at 2 times/day recorded the highest significant FBW, WG, and best FCR among all treatments. The highest significant total biomass weight $\left(\mathrm{kg} \mathrm{hapa}^{-1}\right)$, and gain $\left(\mathrm{kg} \mathrm{hapa}^{-1}\right)$ and feed consumption (kg hapa ${ }^{-}$ ${ }^{1}$ ) were obtained for prawn and tilapia feed the diet containing $35 \%$ protein. No significant differences $(\mathrm{P} \leq 0.05)$ were observed for $\mathrm{SGR}, \mathrm{FCR}$ and $\mathrm{S}$ among all experimental hapas. The same trend was recorded for the different feeding frequencies. The highest values of the whole body CP, EE, ash and gross energy content were recorded for prawn fed on 30\% diet at twice per day. No clear trend was observed for body composition of the Nile tilapia fry except for the highest lipid content $(\mathrm{P} \leq 0.05)$ recorded for the fish fed on $30 \% \mathrm{CP}$ diet 2 times per day. The findings of the current trials revealed that, feeding frequency has a great effect on growth performance and feed utilization efficiency of the freshwater prawn $M$. rosenbergii PL and the Nile tilapia fry. Feeding frequency 4 times per day for $M$. rosenbergii PL either 2 or 3 times per day for Nile tilapia fry is recommended under similar rearing condition to achieve best performance for both animals.
\end{abstract}

Keywords: Dietary protein, feeding frequency, Macrobrachium rosenbergii, Oreochromus niloticus, fish performance, polyculture. 


\section{INTRODUCTION}

The demand for food including aquatic animals is increasing worldwide. The demand of aquatic products for human consumption will grow to 2.5 million metric tons by 2030 from its present production level of 1 million metric tons (Wijkström, 2003). This goes beyond total capture fisheries supply (400000 metric tons in 2008). The shortfall in supply will largely filled in through aquaculture.

The giant freshwater prawn Macrobrachium rosenbergii has become with the Nile tilapia an important part of polyculture ecosystem in developing countries due to large size attainment, tolerance to water quality changes, ability to cope with handling stress and ability to feed on unconventional feedstuffs (Hossain and Islam, 2006). The Egyptian annual production of freshwater prawn has significantly increased from 600 metric tons (Mt) in 1995 to $5409 \mathrm{Mt}$ in 2008 (FAO, 2010). Tilapia is the most representative variety of freshwater aquaculture crops and one of the most widely cultured species in Egypt. The total aquaculture production of tilapia increased from $24916 \mathrm{Mt}$ in 1990 to 635 $000 \mathrm{in} \mathrm{Mt/} \mathrm{year} \mathrm{in} 2008$ and accounted for $63 \%$ of total fish production (1 008 $000 \mathrm{Mt} /$ year) (GAFRD, 2009).

A successful polyculture system consists of simultaneously rearing two or more species with different feeding habits and space utilization with the goal to maximize production (Zimmerman and New, 2000). Both, Nile tilapia and the freshwater prawn prefer similar temperature ranges, so far are not plagued with major disease problems, and reach market size in Egypt within 6 months of culture (Kabir Chowdhury et al., 2007). Tilapia and prawn have different food and feeding habits. Dos Santos and Valenti (2002) reported that the polyculture of the freshwater prawn, $M$. rosenbergii with the Nile tilapia, O. niloticus, did not affect the production of tilapia. It is known that freshwater prawns feed on benthic organisms (Tidwell et al., 1995), detritus and feces (Zimmerman and New, 2000). In an extensive system, tilapia can filter phytoplankton and zooplankton in the upper water column, meanwhile prawn spends most of the time in the pond bottom grazing on bacterial films on the bottom substrate and on the detritus settling from above. In a more intensive system receiving pelleted feeds, tilapia monopolizes the feed; however some feed particles always get to the bottom where the prawn gets. More importantly, the fecal matter from the tilapia contributes to the detritus rain that supports the prawn feed. Therefore, freshwater prawns benefit directly from tilapia feed wastes and feces, and indirectly from sediment enrichment, stimulating the development of benthic organisms. Tilapia is regarded as an omnivorous species and capable of feeding on benthic and attached algal and detritus aggregates (Azim et al., 2003). Macrobrachium-tilapia polyculture reduces the yield of prawns compared to monoculture, but increases total yield of fish and prawns (Garcia-Perez et al., 2000). Therefore, the polyculture system of $M$. rosenbergii with $O$. niloticus 
provide an addition high-valued to tilapia culture (Keysami et al., 2007) and may provide an opportunity to develop a sustainable aquaculture system. In tilapia-prawn polyculture, total production increased without supplying more feed. Hence, the nutrient input efficiency improved (dos Santos and Valenti, 2002).

Cost of aquaculture feed, which is directly related to the protein content, appears to be the major limit factor influence the economic feasibility of aquatic animal farming. Therefore, expensive dietary protein should be optimally utilized to support rapid growth rather than for maintenance of the cultured animal (Bautista 1986). Currently, much attention has been directed towards the influence of feeding schedule in terms of feeding levels, frequency and time on fish and prawn growth indices (D'Abramo, 2002). In the case of crustaceans, it is generally difficult to measure appropriate feed intake in feeding experiments because of the slow feeding habit of these organisms resulting in the leaching of some nutrients. D'Abramo (2002) showed that the freshwater prawn would feed better during their preferred phase according to their feeding behavior.

Therefore, when prawn was fed at the preferred feeding frequency, an improvement may be shown in feed conversion ratio and growth indices. The utilization of dietary protein for the freshwater prawn can change depending on feeding level and feeding frequency and feeding time of day (Teshima et al., 2001). Teshima and Kanazawa (1987) reported that $36-40 \%$ of given diets for M. rosenbergii was found to be lost in the water and not ingested. Meyers (1987) reported that understanding feeding behavior of cultured species can assist in the development of feeding regimes that reduce the metabolic energy costs in feeding and encourage consumption. Since, feeding regimes (feeding frequency and level) largely influence nutrient utilization of prawn and tilapia, the determination of optimal feeding frequency is needed for maximization of their culture production profit and further in depth information for relevant practical feeding in polyculture is needed to develop sustainable higher growth rate, survival and feed utilization efficiency.

The present study was undertaken to investigate the effect of different dietary protein levels and feeding frequencies on growth performance and feed utilization of the freshwater prawn, Macrobrachium rosenbergii with the Nile tilapia, Oreochromuis niloticus in a polyculture system

\section{MATERIALS AND METHODS}

Experimental aquatic animal and culture technique

Experimental polyculture production trial was carried out in the Fish Research Station, El-Kanater El-Khayria, National Institute of Oceanography and Fisheries (NIOF), Cairo, Egypt. Nile tilapia fry with an average weight of $0.2 \pm 0.05 \mathrm{~g}$ were obtained from El-Arabia hatchery, El-Arabia Company, ElSharkia Governorate, Egypt. Post larvae (PL) of the freshwater prawn, M. rosenbergii with an average weight of $0.2 \pm 0.03 \mathrm{~g}$ were obtained from Saft- 
Khalid hatchery, General Authority for Fish Resources Development, El-Bhira Hatchery, El-Bhira Governorate, Egypt.

Prior to the trial, Nile tilapia fry and prawns PL were acclimated separately to experimental conditions for two weeks in 2 concrete ponds ( $8 \times 4 \times 1 \mathrm{~m}$ each). During the acclimation period, Nile tilapia fry and prawns PL were fed on commercial tilapia diet $(28 \%$ CP purchased from ZooControl Company, Cairo, Egypt), at $10 \%$ of body weight daily. The daily ration was divided into two equal portions, fed at 08.00 and 12.00 hours, respectively. Water temperature was recorded daily using a thermometer suspended in each hapa. Dissolved oxygen was measured using a YSI model 56 oxygen meter (YSI Company, Yellow Springs Instrument, Yellow Springs, OH, USA) and $\mathrm{pH}$ using a $\mathrm{pH}$ meter (Orion pH meter, Abilene, TX, USA). Ammonia and salinity were measured two times a week according to Standard Methodology of APHA, AWWA, WPCF (1985).

Six different treatments were assigned in triplicates using two dietary protein levels; 30 and $35 \% \mathrm{CP}$ and three feeding frequencies 2,3 and 4 times a day (8:0 and $11.0 \mathrm{~h}),(8.0,11.0$ and $14.0 \mathrm{~h})$ and $(8.0,11.0,14.0$ and $17.0 \mathrm{~h})$, respectively in a factorial design to determine its influences on growth performance and feed utilization of polyculture the freshwater prawn, $M$. rosenbergii PL and Nile tilapia, O. niloticus fry cultured in small scale hapas. The experiment was conducted in small scale hapas $\left(3.75 \mathrm{~m}^{3}\right.$ each with effective dimension $(\mathrm{L} \times \mathrm{W}$ $\times \mathrm{H}$ ) of $1.25 \times 2.0 \times 1.5 \mathrm{~m}$, respectively). Each hapa was provided with four 30 $\mathrm{cm}$ long $16 \mathrm{~mm}$ diameter black polyvinyl chloride (PVC) pipes to minimize the cannibalism during the molting of prawn (Mariappan and Balasundaram 2004). All hapas were put in running earthen pond system with average volume of 2000 $\mathrm{m}^{3}$ water and $1.5 \mathrm{~m}$ depth. The pond was supplied with fresh water from the Darawa Irrigation Branch, Kalubiya, Governorate. The turnover rate of water was $0.7 \mathrm{~m}^{-3} /$ pond $^{-1} /$ day. Prawns and Nile tilapia were held under natural $12: 12 \mathrm{~h}$ light: dark schedule. 150 freshwater prawns PL with an average weight of $0.2 \pm$ $0.05 \mathrm{~g}$ and 37 Nile tilapia fry with an average body weight of $0.2 \pm 0.02 \mathrm{~g}$ were stocked in each hapa based on the hapa net enclosures bottom area $\left(2.5 \mathrm{~m}^{-2}\right)$ according to FAO (2002). Mortality and limbless were recorded at $08.00 \mathrm{~h}$, every day.

\section{Experimental diets}

Two isocaloric (19.4 $\mathrm{MJ} \mathrm{GE} \mathrm{kg}^{-1}$ ) experimental diets were formulated to contain 30 or $35 \% \mathrm{CP}$, respectively (Table 1). The feed ingredients were ground to powder using a homogenous mixture grinder (Philips, Mode HL1616 ID, Philips India, Calcutta, India), until passing through a $1.0 \mathrm{~mm}$ screen. Diets were processed by blending the dry ingredients into a homogenous mixture, and then the mixture was passed through Sprout-Waldron laboratory pellet mill (CPM, California Pellet Mill Co., San Francisco, California, CA, USA) to form sinking pellets $1 \mathrm{~mm}$ dimensions. Diets were stored in plastic bags at $-4^{\circ} \mathrm{C}$. Freshwater prawns and Nile tilapia were fed daily at level of $5 \%$ for total hapa biomass 6 
days a week for 84 days. The daily ration was fed in equal amounts at 3 feeding frequency: 2, 3 and 4 times a day (8:0 and $11.0 \mathrm{~h}),(8.0,11.0$ and $14.0 \mathrm{~h})$ and $(8.0,11.0,14.0$ and $17.0 \mathrm{~h})$, respectively. In each hapa, during the feeding period, the total weight and number of prawns and Nile tilapia were measured every 2 weeks, to adjust the daily feed ration. Gross energy content of diets were calculated according to caloric values (Brett, 1973) using the values 23.6, 39.5 and $17.2 \mathrm{~kJ} / \mathrm{g}$ for crude protein, crude fat and total carbohydrate, respectively.

Table 1: Formulation $\left(\mathrm{g} \mathrm{kg}^{-1}\right)$ and proximate composition $(\% \mathrm{DM})$ of the experimental diets as-fed-basis.

\begin{tabular}{lcc}
\hline & \multicolumn{2}{c}{ Experimental diets } \\
Ingredients $\left(\mathrm{g} \mathrm{kg}^{-1} / \mathrm{diet}\right)$ & Diet $_{1}$ & Diet $_{2}$ \\
\hline Fish meal & 10 & 10 \\
Poultry by-product meal & 21 & 24 \\
Soybean meal & 17 & 27 \\
Wheat bran & 22 & 17 \\
Yellow corn & 20 & 12 \\
Soybean oil & 4 & 4 \\
Linseed oil & 4 & 4 \\
Corn starch & 0.5 & 0.5 \\
Vitamins \& Mineral premix & 1.5 \\
Proximate composition (\%DM) & 1.5 & 1.5 \\
Moisture & & \\
Crude protein $(\mathrm{CP})$ & 9.0 & 9.4 \\
Crude lipid (CL) & 30.4 & 35.0 \\
Ash & 14.3 & 14.4 \\
Total carbohydrate ${ }^{2}$ & 7.8 & 9.3 \\
Gross energy $(\mathrm{KJ} / \mathrm{kg})^{3}$ & 38.5 & 31.9 \\
\hline
\end{tabular}

${ }^{\mathrm{T}}$ Vitamin and mineral mixture each 1-kg of mixture contains: 4800 I.U. Vit A, 2400 IU cholecalciferol (vit. D) , $40 \mathrm{~g}$ Vit E, $8 \mathrm{~g}$ Vit K, $4.0 \mathrm{~g}$ Vit B $12,4.0 \mathrm{~g}$ Vit B2, $6 \mathrm{~g}$ Vit B6, $4.0 \mathrm{~g}$ Pantothenic acid, $8.0 \mathrm{~g}$ Nicotinic acid, $400 \mathrm{mg}$ Folic acid, $20 \mathrm{mg}$ Biotin, $200 \mathrm{gm}$ Choline, $4 \mathrm{~g}$ Copper, $0.4 \mathrm{~g}$ Iodine, $12 \mathrm{~g}$ Iron, $22 \mathrm{~g}$ Manganese, $22 \mathrm{~g}$ Zinc, $0.04 \mathrm{~g}$ Selenium, $1.2 \mathrm{mg}$ Niacin, 12 $\mathrm{mg}$ D-calcium Pantothenate, $26 \mathrm{mg}$ Pyridoxine. $\mathrm{HCl}, 6 \mathrm{mg}$ Riboflavin, $7.2 \mathrm{mg}$ Thiamin. $\mathrm{HCl}, 1.2$ mg Sodium chloride $(\mathrm{NaCl}, 39 \% \mathrm{Na}, 61 \% \mathrm{Cl}), 3077 \mathrm{mg}$ Ferrous sulfate $\left(\mathrm{FeSO}_{4} .7 \mathrm{H}_{2} \mathrm{O}, 20 \% \mathrm{Fe}\right)$, $65 \mathrm{mg}$ Manganese sulfate $\left(\mathrm{MnSO}_{4}, 36 \% \mathrm{Mn}\right), 89 \mathrm{mg} \mathrm{Zinc}$ sulfate $\left(\mathrm{ZnSO}_{4} \cdot 7 \mathrm{H}_{2} \mathrm{O}, 40 \% \mathrm{Zn}\right), 150$ mg Copper sulfate $\left(\mathrm{CuSO}_{4} .5 \mathrm{H}_{2} \mathrm{O}, 25 \% \mathrm{Cu}\right.$ ), $28 \mathrm{mg}$ Potassium iodide (KI, $24 \% \mathrm{~K}, 76 \% \mathrm{I}$ ), 1000 mg Celite AW521 (acid-washed diatomaceous earth silica).

${ }^{2}$ Total carbohydrates calculated by differences.

${ }^{3}$ Calculated as $23.63,39.52$ and $17.15 \mathrm{~kJ} \mathrm{~g}^{-1}$ for protein, fat and carbohydrate, respectively (Brett 1973.

\section{Growth indices}

The mean final weight of individual prawn PL, Nile tilapia fry and total biomass $(\mathrm{kg})$ per hapa were determined by dividing the total weight by the number of animals. The first sampling was conducted on the $30^{\text {th }}$ day after stocking and at 15 days intervals thereafter. Survival (S), weight gain (WG), Specific growth rate (SGR), feed conversion ratio (FCR), protein efficiency ratio 
(PER), protein productive value (PPV), energy Retention (ER) and economical conversion rate were all calculated using the following equations:

Percentage Survival $(\mathrm{S})=$ (final number of animals / initial number of animals) $\times 100$.

$\mathrm{WG}=$ Final weight $(\mathrm{g})$ - Initial weight $(\mathrm{g})$.

$\mathrm{SGR}=(\ln \mathrm{FBW}-\ln \mathrm{IBW}) / \mathrm{t} \times 100$

Where FBW is the final weight (g); IBW is the initial weight $(\mathrm{g})$; $\ln$ is the natural logarithmic; $t$ is the time in days.

PER $=$ Weight gain $(\mathrm{g}) /$ Protein feed $(\mathrm{g})$.

$\mathrm{PPV}=($ Protein gain $(\mathrm{g}) /$ Protein feed $(\mathrm{g})) \times 100$.

$\mathrm{ER}=($ Energy gain $(\mathrm{kJ}) /$ Energy feed $(\mathrm{kJ})) \times 100$.

$\mathrm{FCR}=$ Total weight of feed consumed (dry) / Wet biomass gain .

\section{Analytical methods}

At end of experimental, 40 prawns PL were randomly selected from each hapa to determine the proximate whole body composition. Per hapa, each animal were blended, homogenized, oven-dried $\left(70{ }^{\circ} \mathrm{C} 48 \mathrm{~h}\right.$ ), grounded and stored at $-20{ }^{\circ} \mathrm{C}$ for subsequent analysis. The proximate composition of each animals and diets samples were determined according to the Association of Official Analytical Chemists (AOAC 1995). Moisture was determined after oven drying $\left(105^{\circ} \mathrm{C}\right)$ for $24 \mathrm{~h}$ (MEMMERT Drying Oven, GE-174, Memmert GmbH, Germany). Ash was measured by incineration at $550^{\circ} \mathrm{C}$ for $12 \mathrm{~h}$ (Thermo Scientific Heraeus M 110 Muffle furnace, Thermo Fisher Scientific, Waltham, MA, USA). Crude protein was determined by the micro-kjeldhal method, $\mathrm{N} \% \times$ 6.25 (using a kjeltech autoanalyzer, Model 1030, Tecator, Hoganas, Sweden) and crude fat by soxhlet extraction with diethyl ether $\left(40-60^{\circ} \mathrm{C}\right)$ (Soxtec System HT6, Tecator).

\section{Statistical analysis}

Results were statistically analyzed with a two-ways analysis of variance (ANOVA) using MSTAT-C Version 4.0 Software Package (MSTAT-C 1987) using Factorial design $(2 \times 3)$. Duncan's multiple range tests was used to compare differences between treatment means when significance $F$ values were observed $(\mathrm{P} \leq 0.05)$ (Duncan 1955). All percentage and ratio data were arc-sin transformed before analysis (Zar 1984). Non-transformed data are presented in Tables to simplify comparisons.

\section{RESULTS}

Water quality parameters during the 84 day experimental period were recorded to be normal for all hapas and remained within ranges allowing for high growth rate and production for either freshwater prawns or Nile tilapia .Water temperature ranged between $27.5 \& 28.8 \pm 0.9^{\circ}$ dissolved oxygen from $\mathrm{C} C$ 5.0 to $6.1 \pm 0.8 \mathrm{mg} \mathrm{L}^{-1}, \mathrm{pH}$ from 6.9 to $7.5 \pm 0.3$ and ammonia from 0.24 to $0.27 \pm 0.1 \mathrm{mg} \mathrm{L}^{-1}$. 
The growth performance and feed utilization efficiency indices for $M$. rosenbergii $\mathrm{PL}$ and O. niloticus fry are shown in (Table 2). Irrespective of polyculture system, prawn $\mathrm{PL}$ fed on diet containing 35\% CP with feeding frequency (4 times a day) had the highest significant $(\mathrm{P} \leq 0.05) \mathrm{FBW}, \mathrm{WG}, \mathrm{SGR}$ and best $\mathrm{FCR}$ values among all treatments. Meanwhile, Nile tilapia fed diet containing 35\% CP with feeding frequency (2 times a day) recorded the highest significant FBW, WG, and best FCR among all treatments.

Table 2: Growth and feed utilization indices and survival (\%) for freshwater prawn $\mathrm{M}$. rosenbergii and Nile tilapia $O$. niloticus under different feeding regimes.

\begin{tabular}{|c|c|c|c|c|c|c|c|c|}
\hline & $\begin{array}{l}\text { Protein levels } \\
\%\end{array}$ & $\begin{array}{c}\text { Feeding frequency } \\
\text { time/day }\end{array}$ & FBW (g) & WG (g) & SGR & FI & FCR & S (\%) \\
\hline \multicolumn{9}{|l|}{ M. rosenbergit } \\
\hline & 30 & 2 & $7.11 \pm 0.95^{\circ}$ & $6.91 \pm 0.94 b$ & $4.25 \pm 0.30 \mathrm{~b}$ & $12.86 \pm 1.85 b$ & $1.84 \pm 0.13 b$ & $47.00 \pm 3.55$ \\
\hline & & 3 & $6.96 \pm 0.65 \mathrm{~b}$ & $6.76 \pm 0.65 \mathrm{~b}$ & $4.23 \pm 0.15 \mathrm{~b}$ & $12.43 \pm 1.22 \mathrm{~b}$ & $1.84 \pm 0.20 \mathrm{~b}$ & $54.00 \pm 6.66$ \\
\hline & & 4 & $6.38 \pm 0.11 \mathrm{~d}$ & $6.18 \pm 0.14 \mathrm{~d}$ & $4.12 \pm 0.09 \mathrm{c}$ & $11.05 \pm 1.66 \mathrm{c}$ & $1.79 \pm 0.24 c$ & $49.00 \pm 3.80$ \\
\hline & 35 & 2 & $6.76 \pm 0.12 c$ & $6.56 \pm 0.12 c$ & $4.19 \pm 0.40 \mathrm{c}$ & $12.69 \pm 1.12 b$ & $1.93 \pm 0.17 \mathrm{a}$ & $47.00 \pm 3.77$ \\
\hline & & 3 & $7.66 \pm 0.11 \mathrm{~b}$ & $7.46 \pm 0.13 b$ & $4.34 \pm 0.80 \mathrm{~b}$ & $11.60 \pm 1.45 \mathrm{c}$ & $1.55 \pm 0.19 \mathrm{~d}$ & $48.00 \pm 7.80$ \\
\hline & & 4 & $8.95 \pm 0.42 a$ & $8.75 \pm 0.24 a$ & $4.53 \pm 0.17 \mathrm{a}$ & $13.37 \pm 1.23 \mathrm{a}$ & $1.53 \pm 0.27 \mathrm{~d}$ & $40.00 \pm 7.10$ \\
\hline \multicolumn{9}{|l|}{ Protein levels } \\
\hline 30 & & & $6.82 \pm 0.35^{\circ}$ & $6.62 \pm 0.40^{b}$ & $4.20 \pm 0.21^{\circ}$ & $12.11 \pm 1.36^{\circ}$ & $1.82 \pm 0.50^{2}$ & $50.00 \pm 6.80^{2}$ \\
\hline 35 & & & $7.79 \pm 0.11^{\mathrm{a}}$ & $7.59 \pm 0.13^{4}$ & $4.35 \pm 0.26^{2}$ & $12.55 \pm 1.02^{\mathrm{a}}$ & $1.67 \pm 0.21^{6}$ & $45.00 \pm 8.00^{6}$ \\
\hline \multicolumn{9}{|c|}{ Feeding frequency } \\
\hline $2=2$ & & & $6.94 \pm 0.23^{6}$ & $6.74 \pm 0.25^{b}$ & $4.22 \pm 0.11^{\circ}$ & $12.78 \pm 1.21^{4}$ & $1.89 \pm 0.16^{6}$ & $47.00 \pm 8.6^{\circ}$ \\
\hline 3 & & & $7.31 \pm 0.22^{b}$ & $7.11 \pm 0.26^{6}$ & $4.29 \pm 0.14^{6}$ & $12.02 \pm 1.55^{6}$ & $1.70 \pm 0.21^{6}$ & $51.00 \pm 5.6^{2}$ \\
\hline 4 & & & $7.67 \pm 0.12^{\mathrm{s}}$ & $7.47 \pm 0.14^{2}$ & $4.33 \pm 0.11^{2}$ & $12.21 \pm 1.86^{\circ}$ & $1.66 \pm 0.70^{4}$ & $44.5 \pm 7.70^{\circ}$ \\
\hline \multicolumn{9}{|l|}{ O. niloticus } \\
\hline & 30 & 2 & $63.10 \pm 6.88 c$ & $62.90 \pm 1.90 \mathrm{c}$ & $6.85 \pm 0.38$ & $97.05 \pm 2.12 c$ & $1.48 \pm 0.14 c$ & $95.00 \pm 1.10$ \\
\hline & & 3 & $70.70 \pm 1.86 \mathrm{~b}$ & $70.50 \pm 1.81 \mathrm{~b}$ & $6.99 \pm 0.70$ & $106.22 \pm 1.22 \mathrm{~b}$ & $1.48 \pm 0.11 \mathrm{c}$ & $96.00 \pm 0.80$ \\
\hline & & 4 & $60.35 \pm 1.55 \mathrm{~d}$ & $60.15 \pm 1.50 \mathrm{~d}$ & $6.79 \pm 0.29$ & $85.01 \pm 1.23 \mathrm{~d}$ & $1.41 \pm 0.21 \mathrm{~d}$ & $91.00 \pm 0.50$ \\
\hline & 35 & 2 & $75.40 \pm 2.22 \mathrm{a}$ & $75.29 \pm 2.02 \mathrm{a}$ & $7.06 \pm 0.36$ & $106.13 \pm 2.35 b$ & $1.41 \pm 0.32 \mathrm{~d}$ & $93.00 \pm 0.10$ \\
\hline & & 3 & $71.10 \pm 2.02 b$ & $70.90 \pm 2.00 \mathrm{~b}$ & $6.68 \pm 0.50$ & $108.81 \pm 1.42 \mathrm{a}$ & $1.54 \pm 0.23 b$ & $90.00 \pm 2.00$ \\
\hline & & 4 & $64.11 \pm 2.36 c$ & $63.19 \pm 2.21 \mathrm{c}$ & $6.87 \pm 0.27$ & $99.57 \pm 1.12 \mathrm{c}$ & $1.58 \pm 0.32 \mathrm{a}$ & $91.00 \pm 1.50$ \\
\hline \multicolumn{9}{|l|}{ Protein levels } \\
\hline 30 & & & $64.72 \pm 1.23^{\circ}$ & $64.52 \pm 1.13^{b}$ & $6.88 \pm 0.37^{6}$ & $96.09 \pm 2.65^{6}$ & $1.46 \pm 0.14^{\circ}$ & $94.00 \pm 0.8$ \\
\hline 35 & & & $70.20 \pm 1.45^{2}$ & $69.79 \pm 1.30^{2}$ & $6.98 \pm 0.42^{\mathrm{a}}$ & $104.84 \pm 1.95^{2}$ & $1.51 \pm 0.30^{4}$ & $91.33 \pm 0.70$ \\
\hline \multicolumn{9}{|c|}{ Feeding frequency } \\
\hline 2 & & & $69.25 \pm 1.12^{\mathrm{a}}$ & $69.10 \pm 1.02^{\mathrm{s}}$ & $6.96 \pm 0.42^{26}$ & $101.59 \pm 1.53^{\circ}$ & $1.45 \pm 0.14$ & $94.00 \pm 1.25$ \\
\hline 3 & & & $70.90 \pm 0.66^{2}$ & $70.70 \pm 0.60^{2}$ & $6.84 \pm 0.21^{\mathrm{a}}$ & $107.52 \pm 1.42^{c}$ & $1.51 \pm 0.21$ & $93.00 \pm 1.50$ \\
\hline 4 & & & $62.23 \pm 1.22^{6}$ & $61.67 \pm 1.22^{6}$ & $6.83 \pm 0.23^{\circ}$ & $92.29 \pm 2.23^{c}$ & $1.50 \pm 0.32$ & $91.00 \pm 0.50$ \\
\hline
\end{tabular}

Means values $( \pm \mathrm{SD})$ in the same column sharing the same superscripts (within each species) are not significant different $(\mathrm{P} \leq 0.05)$

No significant difference $(\mathrm{P} \leq 0.05)$ was observed for SGR of Nile tilapia fry among all experimental treatments. The same trend was recorded for $\%$ survival prawns PL (S \%). Considering the hapa biomass $\left(\mathrm{kg} \mathrm{hapa}^{-1}\right)$ (Table 3), the highest significant values of total biomass weight $\left(\mathrm{kg} \mathrm{hapa}^{-1}\right)$, total biomass gain $\left(\mathrm{kg} \mathrm{hapa}^{-1}\right)$ and feed intake $\left(\mathrm{kg} \mathrm{hapa}^{-1}\right)$ were observed for both cultured animals fed the diet containing $35 \%$ protein.

Table 3: Effect of different dietary protein levels and feeding frequency on average final biomass and feed conversion ratio of freshwater prawn, $M$. rosenbergii and Nile tilapia, $O$. niloticus in Polyculture.

\begin{tabular}{|c|c|c|c|c|c|c|c|c|}
\hline $\begin{array}{l}\text { Protein } \\
\text { levels \% }\end{array}$ & $\begin{array}{c}\text { Feeding frequency } \\
\text { time/day }\end{array}$ & $\begin{array}{c}\text { Initial biomass } \\
\text { (kg/hapa) }\end{array}$ & $\begin{array}{c}\text { Final biomass } \\
\text { (kg/hapa) }\end{array}$ & $\begin{array}{c}\text { Gain } \\
\text { (kg/hapa) }\end{array}$ & FC & SGR & FCR & $\mathbf{S}(\%)$ \\
\hline \multirow[t]{3}{*}{30} & 2 & $0.04 \pm 0.02$ & $2.72 \pm 0.25 \mathrm{c}$ & $2.68 \pm 0.24 c$ & $4.32 \pm 0.15$ & $5.10 \pm 0.28$ & $1.61 \pm 0.31$ & $71 \pm 2.12$ \\
\hline & 3 & $0.04 \pm 0.02$ & $3.08 \pm 0.37 \mathrm{a}$ & $3.04 \pm 0.35 \mathrm{a}$ & $4.78 \pm 0.15$ & $5.25 \pm 0.53$ & $1.57 \pm 0.20$ & $75 \pm 3.11$ \\
\hline & 4 & $0.04 \pm 0.02$ & $2.50 \pm 0.11 \mathrm{~d}$ & $2.46 \pm 0.12 \mathrm{~d}$ & $3.68 \pm 0.36$ & $5.37 \pm 0.50$ & $1.49 \pm 0.24$ & $70 \pm 2.20$ \\
\hline \multirow[t]{3}{*}{35} & 2 & $0.04 \pm 0.02$ & $3.07 \pm 0.42 a$ & $3.03 \pm 0.41 \mathrm{a}$ & $4.55 \pm 0.42$ & $5.25 \pm 0.63$ & $1.50 \pm 0.28$ & $70 \pm 1.97$ \\
\hline & 3 & $0.04 \pm 0.02$ & $2.92 \pm 0.21 \mathrm{~b}$ & $2.88 \pm 0.20 b$ & $4.46 \pm 0.65$ & $5.19 \pm 0.45$ & $1.55 \pm 0.22$ & $69 \pm 3.11$ \\
\hline & 4 & $0.04 \pm 0.02$ & $2.70 \pm 0.42 c$ & $2.66 \pm 0.40 \mathrm{c}$ & $4.16 \pm 0.29$ & $5.09 \pm 0.46$ & $1.56 \pm 0.38$ & $66 \pm 3.13$ \\
\hline \multicolumn{9}{|c|}{ Protein levels } \\
\hline & 30 & $0.04 \pm 0.02$ & $2.77 \pm 0.24 a$ & $2.73 \pm 0.22 b$ & $4.26 \pm 0.20 \mathrm{~b}$ & $5.24 \pm 0.26$ & $1.56 \pm 0.51$ & $72 \pm 0.26$ \\
\hline & 35 & $0.04 \pm 0.02$ & $2.90 \pm 0.33 \mathrm{~b}$ & $2.86 \pm 0.31 \mathrm{a}$ & $4.39 \pm 0.24 a$ & $5.18 \pm 0.29$ & $1.54 \pm 0.53$ & $68 \pm 0.29$ \\
\hline \multicolumn{9}{|c|}{ Feeding frequency } \\
\hline & 2 & $0.04 \pm 0.02$ & $2.90 \pm 0.29 \mathrm{~b}$ & $2.86 \pm 0.26 b$ & $4.44 \pm 0.29 \mathrm{~b}$ & $5.18 \pm 0.28$ & $1.56 \pm 0.41$ & $71 \pm 0.28$ \\
\hline & 3 & $0.04 \pm 0.02$ & $3.00 \pm 0.31 \mathrm{a}$ & $2.96 \pm 0.22 \mathrm{a}$ & $4.62 \pm 0.32 \mathrm{a}$ & $5.22 \pm 0.20$ & $1.56 \pm 0.45$ & $72 \pm 0.20$ \\
\hline & 4 & $0.04 \pm 0.02$ & $2.60 \pm 0.26 \mathrm{c}$ & $2.56 \pm 0.23 \mathrm{c}$ & $3.92 \pm 0.22 \mathrm{c}$ & $5.17 \pm 0.31$ & $1.53 \pm 0.45$ & $68 \pm 0.31$ \\
\hline
\end{tabular}

Means in the same column with different superscripts (within each species) are significant at a level $(\mathrm{P} \leq 0.05)$ 
No significant differences were observed for SGR, FCR and S among all experimental hapas. The same trend was observed for the different feeding frequencies.

The proximate composition of $M$. rosenbergii and $O$. niloticus are shown in (Table 4). Considering of polyculture system, the highest significant values $(\mathrm{P} \leq$ 0.05) of whole body $\mathrm{CP}, \mathrm{EE}$, ash and gross energy content were recorded for prawn PL fed diet content $30 \%$ at feeding frequency of 2 times a day. Regarding Nile tilapia, no clear trend was observed for whole body composition of fry except for the highest lipid content recorded for fish fed the diet containing $30 \%$ CP 2 times a day. No significant difference were shown for all major nutrients measured as the effect of either different dietary protein level or feeding frequency, irrespective of polyculture system for both $M$. rosenbergii PL and Nile tilapia fry .

Table 4: Effect of different dietary protein levels and feeding frequencies on the proximate composition (wet weight basis) of freshwater prawn $M$. rosenbergii and Nile tilapia $O$. niloticus fed different feeding regimes.

\begin{tabular}{|c|c|c|c|c|c|c|}
\hline Dietary Protein levels (\%) & $\begin{array}{c}\text { Feeding frequency } \\
\text { time/day }\end{array}$ & Crude Protein & Ether extract & Ash & Moisture & $\begin{array}{l}\text { Gross energy } \\
\text { (MJ/100g) }\end{array}$ \\
\hline \multicolumn{7}{|l|}{ M. rosenbergii } \\
\hline \multirow[t]{3}{*}{30} & 2 & $19.87 \pm 0.28^{4}$ & $4.61 \pm 0.13^{2}$ & $5.02 \pm 0.16^{2}$ & $73.50 \pm 0.68$ & $580.80 \pm 12.50^{2}$ \\
\hline & 3 & $15.17 \pm 0.44^{c}$ & $3.98 \pm 0.20^{b}$ & $3.71 \pm 0.14^{c}$ & $77.14 \pm 0.10$ & $515.73 \pm 10.11^{\circ}$ \\
\hline & 4 & $16.29 \pm 0.02^{b}$ & $3.08 \pm 0.52^{4}$ & $4.74 \pm 0.30^{6}$ & $75.89 \pm 0.20$ & $506.63 \pm 8.60^{4}$ \\
\hline \multirow[t]{3}{*}{35} & 2 & $15.18 \pm 0.46^{6}$ & $3.86 \pm 0.80^{6}$ & $4.51 \pm 5.15^{6}$ & $76.45 \pm 0.16$ & $511.22 \pm 24.50^{6}$ \\
\hline & 3 & $15.79 \pm 0.35^{\circ}$ & $3.82 \pm 0.27^{\mathrm{c}}$ & $3.71 \pm 0.44^{a}$ & $76.68 \pm 0.11$ & $524.06 \pm 26.11^{6}$ \\
\hline & 4 & $15.99 \pm 0.42^{b}$ & $3.70 \pm 0.12^{c}$ & $2.89 \pm 0.17^{\mathrm{d}}$ & $77.42 \pm 0.30$ & $524.04 \pm 18.11^{\circ}$ \\
\hline \multicolumn{2}{|l|}{ Protein level } & & & & & \\
\hline 30 & & $16.11 \pm 0.16$ & $3.89 \pm 0.42$ & $4.49 \pm 0.98$ & $75.51 \pm 0.70$ & $543.39 \pm 21.11$ \\
\hline 35 & & $15.65 \pm 0.12$ & $3.79 \pm 0.30$ & $3.70 \pm 0.80$ & $76.85 \pm 0.50$ & $519.77 \pm 16.14$ \\
\hline \multicolumn{2}{|l|}{ Feeding frequency } & & & & & \\
\hline 2 & & $16.03 \pm 0.20$ & $4.24 \pm 0.40$ & $4.77 \pm 1.10$ & $74.98 \pm 0.69$ & $546.81 \pm 10.80$ \\
\hline 3 & & $15.18 \pm 0.18$ & $3.90 \pm 0.30$ & $3.71 \pm 0.88$ & $76.19 \pm 0.50$ & $520.17 \pm 10.20$ \\
\hline 4 & & $16.14 \pm 0.20$ & $3.39 \pm 0.48$ & $3.82 \pm 0.80$ & $76.66 \pm 0.40$ & $515.34 \pm 21.81$ \\
\hline \multicolumn{7}{|l|}{ O. niloticus } \\
\hline \multirow[t]{3}{*}{30} & 2 & $15.49 \pm 0.30$ & $5.34 \pm 0.15^{4}$ & $5.22 \pm 0.80$ & $73.95 \pm 0.65$ & $577.04 \pm 12.60$ \\
\hline & 3 & $15.75 \pm 0.32$ & $5.03 \pm 0.40^{3 b}$ & $5.42 \pm 0.98$ & $73.80 \pm 0.11$ & $571.93 \pm 8.60$ \\
\hline & 4 & $14.81 \pm 0.31$ & $4.47 \pm 0.17^{6}$ & $5.04 \pm 1.11$ & $75.68 \pm 0.31$ & $526.59 \pm 15.55$ \\
\hline \multirow[t]{3}{*}{35} & 2 & $15.52 \pm 0.23$ & $4.02 \pm 0.21^{\mathrm{G}}$ & $5.15 \pm 2.13$ & $75.31 \pm 0.13$ & $525.58 \pm 18.18$ \\
\hline & 3 & $14.64 \pm 0.32$ & $4.68 \pm 0.32^{\circ}$ & $5.88 \pm 1.60$ & $74.80 \pm 0.42$ & $530.87 \pm 21.10$ \\
\hline & 4 & $14.59 \pm 0.70$ & $4.20 \pm 0.14^{b}$ & $5.11 \pm 2.50$ & $75.74 \pm 0.35$ & $519.23 \pm 12.60$ \\
\hline \multicolumn{2}{|l|}{ Protein level } & & & & & \\
\hline 30 & & $15.02 \pm 0.23$ & $4.95 \pm 0.11$ & $5.23 \pm 1.66$ & $74.48 \pm 0.23$ & $558.19 \pm 18.50$ \\
\hline 35 & & $15.04 \pm 0.32$ & $4.30 \pm 0.36$ & $5.38 \pm 1.86$ & $75.28 \pm 0.22$ & $525.23 \pm 13.60$ \\
\hline \multicolumn{2}{|l|}{ Feeding fiequency } & & & & & \\
\hline 2 & & $15.12 \pm 0.13$ & $5.19 \pm 0.27$ & $5.32 \pm 1.33$ & $73.88 \pm 0.11$ & $573.99 \pm 18.8$ \\
\hline 3 & & $15.17 \pm 0.23$ & $4.25 \pm 0.25$ & $5.10 \pm 1.20$ & $75.50 \pm 0.35$ & $526.09 \pm 13.60$ \\
\hline 4 & & $14.80 \pm 0.32$ & $4.44 \pm 0.33$ & $5.50 \pm 1.60$ & $75.72 \pm 0.22$ & $525.08 \pm 16.15$ \\
\hline
\end{tabular}

Means in the same column with different superscripts (within each species) are significant differences by $(\mathrm{P} \leq 0.05)$.

\section{DISCUSSION}

Water quality parameters during the 84 day experimental period were observed to be certain normal within the ranges for all hapas and remained within these ranges allowing for high growth rate and production for $M$. rosenbergii $\mathrm{PL}$ and $O$. niloticus fry. The higher survival rates shown in the present study may be attributed to the PVC pipes that may have helped the 
prawn to overcome molting stress and to avoid cannibalism (Mariappan and Balasundaram 2004).

Polyculture of prawn with tilapias provide a sustainable aquaculture system (Fitzsimmons, 2001). Giant freshwater prawn M. rosenbergii is an important species and has become an important part of tilapia polyculture ecosystem in developing countries. Nowadays, the polyculture of the freshwater prawn with finfish especially with tilapia is of an enormous interest. In traditional forms of polyculture, waste products of primary species and the natural productivity they foster provide the basis for production of supplemental species. Recently, polyculture has begun to regain attention as a possible mean to increase efficiency in aquaculture production systems, and to reduce environmental impacts caused by excess nutrients. Synergism (some species actually perform better in the presence of other species) is often seen in polyculture systems. Freshwater prawns are a good example. When certain fish species that do not have a particular appetite for prawns, such as tilapia (Martino and Wilson, 1986) are stocked with $M$. rosenbergii, their feces serve as a substrate for bacterial growth, which in turn provides additional nourishment for the prawns. Polyculture can also provide benefits associated with advanced ecological stability and function (McKinnon et al., 2000).

The results of current study have shown that $35 \%$ dietary protein was recommended to achieve the optimum growth for $M$. rosenbergii PL. These results are in agreement with the findings of Teshima et al. (2006) and Kabir Chowdhury et al. (2007) who reported that the increase of body fat content was observed when $M$. rossenbergii PL fed higher dietary protein diets $(>35 \% \mathrm{CP}$ ) as compared to the prawn fed diet of $35 \% \mathrm{CP}$. This is indicative that the increase in dietary protein level exceeding the optimum requirement results in the loss of expensive protein in $M$. rosenbergii diets and accumulation of fats. D'Abramo and Sheen (1994) and Teshima et al. (2006) reported that protein requirement of $M$. rosenbergii PL is considered to be lower than other prawn species and this, may be due to less protein requirement for maintenance compared to carnivorous marine prawn species. Teshima et al. (2001) reported that the optimum dietary protein levels for prawn can change depending on feeding level, feeding frequency, feeding time, sex and other factors such as protein quality, and diet composition.

Recently, much attention has been paid to the influence of feeding schedule in terming of feeding levels and frequency, on fish and prawn growth indices as an important component of successful polyculture system (D'Abramo, 2002). Results of the present study are also in agreement with the findings of Akyiama and Polanco (1997) who recommended four feeding frequencies per day for shrimp. Generally, studies on feeding frequency and its effect on shrimp growth are conflicting. Sedgwick (1979), Robertson et al. (1993) and Tacon et al. (2002) showed a positive effect on shrimp growth when feed frequency was increased. The present result also shows that feeding frequency has a great effect 
on growth performance and feed utilization of Nile tilapia fry. The higher significant $(\mathrm{P} \leq 0.05)$ growth indices for Nile tilapia are observed when fed either 2 or 3 times a day compared to those fed 4 times a day. Mahmoud et al. (2009) showed that growth rates of cultured Nile tilapia was higher at feeding rate of twice a day than feeding once daily. Nguyen and Nguyen (2010) reported that different feeding frequencies (2, 3 \& 4 times per day) had not influenced on mean final weight and feed conversion ratio (FCR) of Nile tilapia O. niloticus juveniles. Meanwhile, when considering, both growth rate (final mean weight) and FCR of Nile tilapia, $O$. niloticus juveniles, it was clear that feeding fish twice a day at $80 \%$ of satiation level had resulted in the highest economic return on investment. On the other hand, Velasco et al. (2000), Smith et al. (2002) and Carvalho and Nunes (2006) found no direct relation between these two factors (dietary protein levels and feeding frequencies). Irrespective of the polyculture system, 35\% dietary protein obtained the optimum growth indices and better FCR for Nile tilapia fry (Table 2). Generally, the FCR was found approximately equal to 2 when shrimp and Nile tilapia culture in low salinity water and polyculture compared to the monoculture (about 1.6).

The results of the present study showed that the highest significant values of total biomass production $\left(\mathrm{kg} \mathrm{hapa}^{-1}\right)$, total biomass gain $\left(\mathrm{kg} \mathrm{hapa}{ }^{-1}\right)$ and feed consumption ( $\mathrm{kg} \mathrm{hapa}^{-1}$ ) were obtained for both cultured animals after being fed on $35 \%$ protein diet or 3 time a day (Table 3). No significant differences were observed for SGR, FCR and $\% \mathrm{~S}$ as an effect of dietary protein content among all experimental hapas. The same trend was observed for the different feeding frequencies. Dos Santos and Valenti (2002) reported that the polyculture system of prawns $M$. rosenbergii with Nile tilapia $O$. niloticus allowed an increase in total production with the same amount of supplied feed, thus improving the system sustainability. However, in the case of crustaceans, it is generally difficult to measure appropriate feed in feeding experiments because of the slow feeding habit of those organisms resulting in the leaching of some nutrients. Meyers (1987) reported that understanding feeding behavior of cultured species can assist in the development of feeds and regimes that reduce the metabolic energy costs in feeding and encourage consumption. Pontes et al. (2008) pointed out that in shrimp farming, feed is commonly offered two or three times per day. Ration is determined by feed tables, which mainly consider stocked shrimp biomass. Rations are also determined through daily recording of uneaten feed left, because this practice does not consider the shrimp physiological factors involved in the feeding, thus inefficient use of the feed may result, with consequent economic and environmental losses.

In conclusion the present results showed that feeding frequency has a great effect on growth performance and feed utilization of freshwater prawn $M$. rosenbergii PL. The highest growth indices for $M$. rosenbergii was observed after being fed 4 times a day compared to Nile tilapia fry fed either 2 or 3 times a day. Irrespective of polyculture system, 35\% protein had led to optimum 
growth indices and better FCR either for freshwater prawn M. rosenbergii PL or Nile tilapia, O. niloticus fry.

\section{REFERENCES}

Akyiama D. and Polanco B. (1997). Semi-Intensive Shimp Farm Management. Technical Manual (ed. by B. Polanco), 30pp. American Soybean Association, Caracas, Venezuela.

AOAC (Association of Official Analytical Chemists) (1995). Official Methods of Analysis. AOAC Inc., 1234 pp, Washington, DC., USA.

APHA, AWWA, WPCF (Standard Methods for the Examination of Water and Wastewater) (1985). 19 ${ }^{\text {th }}$ Edition. American Public Health Association American Water Works Association and Water Pollution Control Federation, Washington, DC, 1268 pp.

Azim, M.E.; Verdegem, M.C.J.; Singh, M.; Van Dam, A. A. and Beveridge, M.C. (2003). The effects of periphyton substrate and fish stocking density on water quality, phytoplankton, periphyton and fish growth. Aquacult. Res., 34: 685-695.

Brett, J.R. (1973). Energy expenditure of Sockeye salmon Oncorhynchus nerka, during sustained performance. J. Fish Res. Board Can., 30 (12/1):1799- 1809.

Bautista, M.N. (1986). The response of Penaeus monodon juveniles to varying protein/energy ratios in test diets. Aquacult., 53 (3-4):229-242

Carvalho, E. A. and Nunes, A. J. P. (2006). Effects of feeding frequency on feed leaching loss and grow-out patterns of the white shrimp Litopenaeus vannamei fed under a diurnal feeding regime in pond enclosures. Aquacult., 252:494-502.

D'Abramo, L. R. (2002). Challenges in developing successful formulated feed for culture of larval fish and crustaceans. In: Avances en Nutrición Acuícola VI. Memorias del VI Simposium Internacional de Nutrición Acuícola. 3 al 6 de Septiembre del 2002 (ed. by L.E. Cruz-Suárez, D. Ricque-Marie, M. Tapia-Salazar, M.G., Gaxiola-Cortés \& N. Simoes). Cancun, Quintana Roo, Mexico pp.143-151.

D’Abramo, L.R. and Sheen, S.S. (1994). Nutritional requirements, feed formulation and feeding practices for intensive culture of the fresh water prawn Macrobrachium rosenbergii. Rev. Fish. Sci., 2:1-21. 
Dos Santos, M.J.M. and Valenti, W.C. (2002). Production of Nile tilapia, Oreochromis niloticus, and freshwater prawn, Macrobrachium rosenbergii, stocked at different densities in polyculture systems in Brazil. Journal of the World Aquacult. Soci., 33(3): 369-376.

Duncan, D.B. (1955). Multiple range and Multiple F test. Biometrics, 11: 1-112.

FAO, (Food and Agricultural Organization) (2002). Farming of Freshwater Prawns: A Manual for the culture of the giant river prawn (Macrobrachium rosenbergii). FAO Fisheries Technical Paper 428. Food and Agriculture Organization, Rome, Italy.

FAO (Food and Agricultural Organization) (2010). Aquaculture Production (Quantities and values) 1950-2008. FishStat Plus-Universal software for fishery statistical time series. Data extracted from the FAO Fisheries Global Aquaculture Production Database for freshwater crustaceans, at: http://www.fao.org/fishery/statistics/software/fishstat/en

Fitzsimmons, K. (2001). Polyculture of tilapia and penaeid shrimp". Global Aquaculture Advocate, 4(3): 43-44.

GAFRD (2009). General Authority for Fish Resources Development. Statistical analysis of total aquaculture production in Egypt. Ministry of Agriculture, Cairo, Egypt (Arabic edition).

Garcia-Perez, A.; Alston, D. and Cortes-Maldonado, R. (2000). Growth, survival, yield and size distribution of freshwater prawn, Macrobrachium rosenbergii, and tilapia, Oreochromis niloticus, in polyculture and monoculture systems in Puerto Rico”. J. Aquacult. Soci., 31(3): 446-451.

Hossain M.A. and Islam M.S. (2006). Optimization of stocking density of freshwater prawn Macrobrachium rosenbergii (de man) in carp polyculture in Bangladesh. Aquacult. Res., 37: 994-1000.

Kabir Chowdhury M.A.; El-Haroun E.R.; Goda A.M.A.-S.; Wafa M.A. and Salah El-Din S.A. (2007) Growth performance of post-larval freshwater prawn Macrobrachium rosenbergii (de Man 1879) at different dietary protein levels and feeding times. Aquaculture 2007, Science for Sustainable Aquaculture, February, 2007, San Antonio Convention Center, San Antonio, Texas, USA, World Aquacult. Soci. Ann. Meeting 2007.

Keysami, M.A.; Saad C.R.; Sijam K.; Daud H.M. and Alimon A.R. (2007). Effect of Bacillus subtitles on growth development and survival of larvae Macrobrachium rosenbergii (deMan). Aquacult. Nut., 13: 131-136. 
Mahmoud, S.H.; Ibrahim, E.M.; Sayed, S.H. and Hassan, A.S. (2009). Effect of feeding frequency on the growth of Nile tilapia (Oreochromis niloticus Lin.) fingerlings and ponds water quality. Egypt. J. Appl. Sci., 24 (1): 31-38.

Mariappan, P. and Balasundaram, C. (2004). Effect of shelters, Densities, and weight Groups on survival, growth and Limb loss in the fresh water prawn, Macrobrachium rosenbergii. J. Appl. Aquacult., 15: 51 - 63.

Martino, C. and Wilson, J. L. (1986). Behavioral interactions of tilapia, crawfish and freshwater prawns in aquaculture. Aquacult. Mag., 12(4):36-37.

McKinnon, L.; Gooley, G.; Ingram, B.; De Silva, S. and Gasior, R. (2000). Directions for integrated aquaculture in Victoria. In: Kumar, M.S. (ed.) Proceedings of National Workshop on Wastewater Treatment and Integrated Aquaculture Production, 17-19 Sept. 1999. SARDI: Henley Beach. 191 pp. O'Connor, J. 1994. Is sustainable capitalism possible? In: O'Connor, M. (ed.) Is capitalism sustainable? Political economy and the politics of ecology. Guilford Press: New York. 283 pp.

Meyers, S.P. (1987). Aquaculture feeds and chemoattractants. Infofish Marketing Digest, 1/87: 35-37.

MSTAT-C Version 4, (1987). Software program for the design and analysis of agronomic research experiments. Michigan State University, Mississippi, MI, USA.

Nguyen, H.M.T. and Nguyen, T.N. (2010). Determination of suitable feeding frequency and ratio for Nile tilapia, Oreochromis niloticus juvenile. Aquaculture 2010, sustainable= profitable, March 1-5, 2010, San Diego, California, USA, World Aquacult. Soc. Ann. Meeting 2010.

Pontes, C. Soares de L.; Patricia P. and Arruda, M. de F. (2008). Feeding responses of juvenile shrimp, Litopenaeus vannamei (Boone) fed at different frequencies under laboratory conditions. Aquacult. Res., 39(13):1416-1422

Robertson, L.; Lawrence A.L. and Castille F.L. (1993). Effect of feeding frequency and feeding time on growth of Penaeus vannamei (Boone). Aquacult. and Fish. Manag., 24:1-6.

Sedgwick R.W. (1979). Effect of ration size and feeding frequency on the growth and food conversion of juvenile Penaeus merguiensis De Man. Aquacult., 16: 279-298. 
Smith D.M.; Burford M.A.; Tabrett S.J.; Irvin S.J. and Ward L. (2002). The effect of feeding frequency on water quality and growth of the black tiger shrimp (Penaeus monodon). Aquacult., 207:125-136.

Tacon A.J.G., Cody J.J., Conquest L.D., Divakaran S., Forster I.P. \& Decamp O.E. (2002) Effect of culture system on the nutrition and growth performance of Pacific white shrimp Litopenaeus vannamei (Boone) fed different diets. Aquacult. Nut., 8:121-137.

Teshima S. and Kanazawa A. (1987). Turnover of dietary cholesterol and bsitosterol in the prawn. Nippon Suisan Gakkaishi, 53: 601-607.

Teshima, S.; Koshio S. and Ishikawa M. (2006). Protein requirements of the freshwater prawn Macrobrachium rosenbergii evaluated by the factorial method. J. World Aquacult. Soci., 37(2): 145-153.

Teshima, S.; Koshio, S.; Ishikawa, M. and Kanazawa, A. (2001). Protein requirement of the prawn Marsupenaeus japonicus estimated by a factorial method. Hydrobiologia, 449: 293-300.

Tidwell, J.H.; Webster, C.D.; Sedlacek, J.D.; Weston, P.A.; Knight, W.L.; Hill Jr., S.J.; D’Abramo, L.R.; Daniels, W.H.; Fuller, M.J. and Montafiez, J.L. (1995). Effects of complete and supplemental diets and organic pond fertilization on production of Macrobrachium rosenbergii and associated benthic macroinvertebrate populations. Aquacult., 138: 169-180.

Velasco, M.; LawrenceA, L.; Castille, F.L. and Obaldo L.G. (2000). Dietary protein requirement for Litopenaeus vannamei. In: Proceedings of the $\mathrm{V}$ International Symposium on Aquatic Nutrition, Advances en Nutrition Acucola V (ed. by L.E. Cruz-Suarez, D. Ricque-Marie, M. Tapia- Salazar, M. Olvera-Novoa \& R. Civera-Cerecedo), pp. 181-192. Universidad Autonoma de Nuevo Leon, Nuevo Leon, Mexico.

Wijkström, U.N. (2003). Short and long-term prospects for consumption of fish. Veterinary Research Communications, 27 (1): 461-468.

Zar J.H. (1984). Biostatistical Analysis. Prentice-Hall, Englewood Cliff, NJ, USA.

Zimmerman, S. and New, M.B. (2000). Grow-out system-poly-culture and integrated culture. In: Freshwater Prawn Culture: The Farming of Macrobrachium rogenbergii (ed. by M.B. New and W.C. Valenti) pp. 187202, Blackwell Science, Oxford, UK. 